
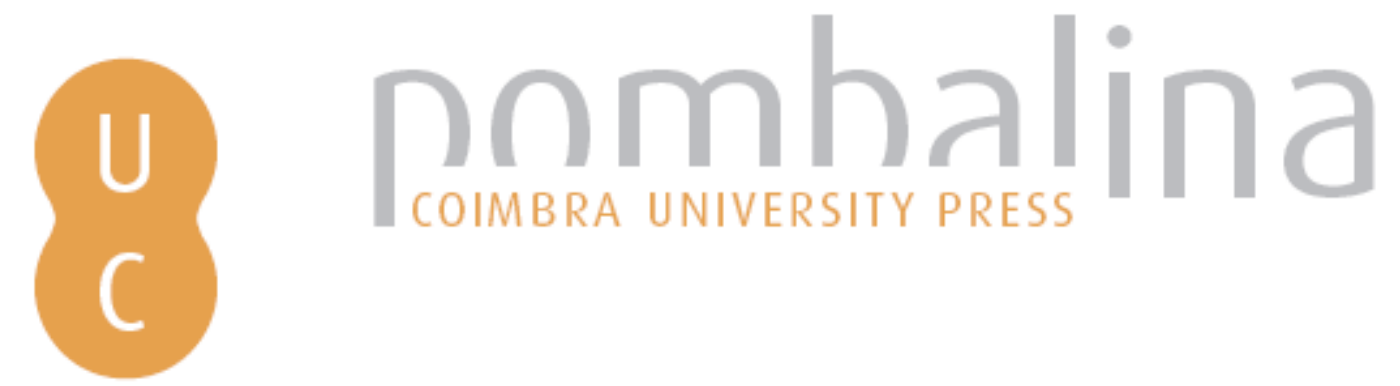

\title{
Éros, Arès et Aphrodite dans l' Érotikos: une reeonsidération de la réponse à Pemptides (ch. 13-18)
}

\author{
Autor(es): $\quad$ Frazier, Françoise
}

Publicado por: Imprensa da Universidade de Coimbra; Katholieke Universiteit Leuven

URL

persistente: URI:http://hdl.handle.net/10316.2/32100

DOI: $\quad$ DOI:http://dx.doi.org/10.14195/978-989-26-0462-6_9

Accessed : $\quad$ 26-Apr-2023 11:05:38

A navegação consulta e descarregamento dos títulos inseridos nas Bibliotecas Digitais UC Digitalis, UC Pombalina e UC Impactum, pressupõem a aceitação plena e sem reservas dos Termos e Condições de Uso destas Bibliotecas Digitais, disponíveis em https://digitalis.uc.pt/pt-pt/termos.

Conforme exposto nos referidos Termos e Condições de Uso, o descarregamento de títulos de acesso restrito requer uma licença válida de autorização devendo o utilizador aceder ao(s) documento(s) a partir de um endereço de IP da instituição detentora da supramencionada licença.

Ao utilizador é apenas permitido o descarregamento para uso pessoal, pelo que o emprego do(s) título(s) descarregado(s) para outro fim, designadamente comercial, carece de autorização do respetivo autor ou editor da obra.

Na medida em que todas as obras da UC Digitalis se encontram protegidas pelo Código do Direito de Autor e Direitos Conexos e demais legislação aplicável, toda a cópia, parcial ou total, deste documento, nos casos em que é legalmente admitida, deverá conter ou fazer-se acompanhar por este aviso.

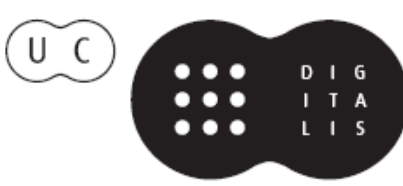




\title{
Puilosophy in Society \\ Virtues and Values in Plutarch
}

\author{
José Ribeiro Ferreira \\ LUC VAN DER STOCKT \\ Maria do Céu Fialho
}

Editors

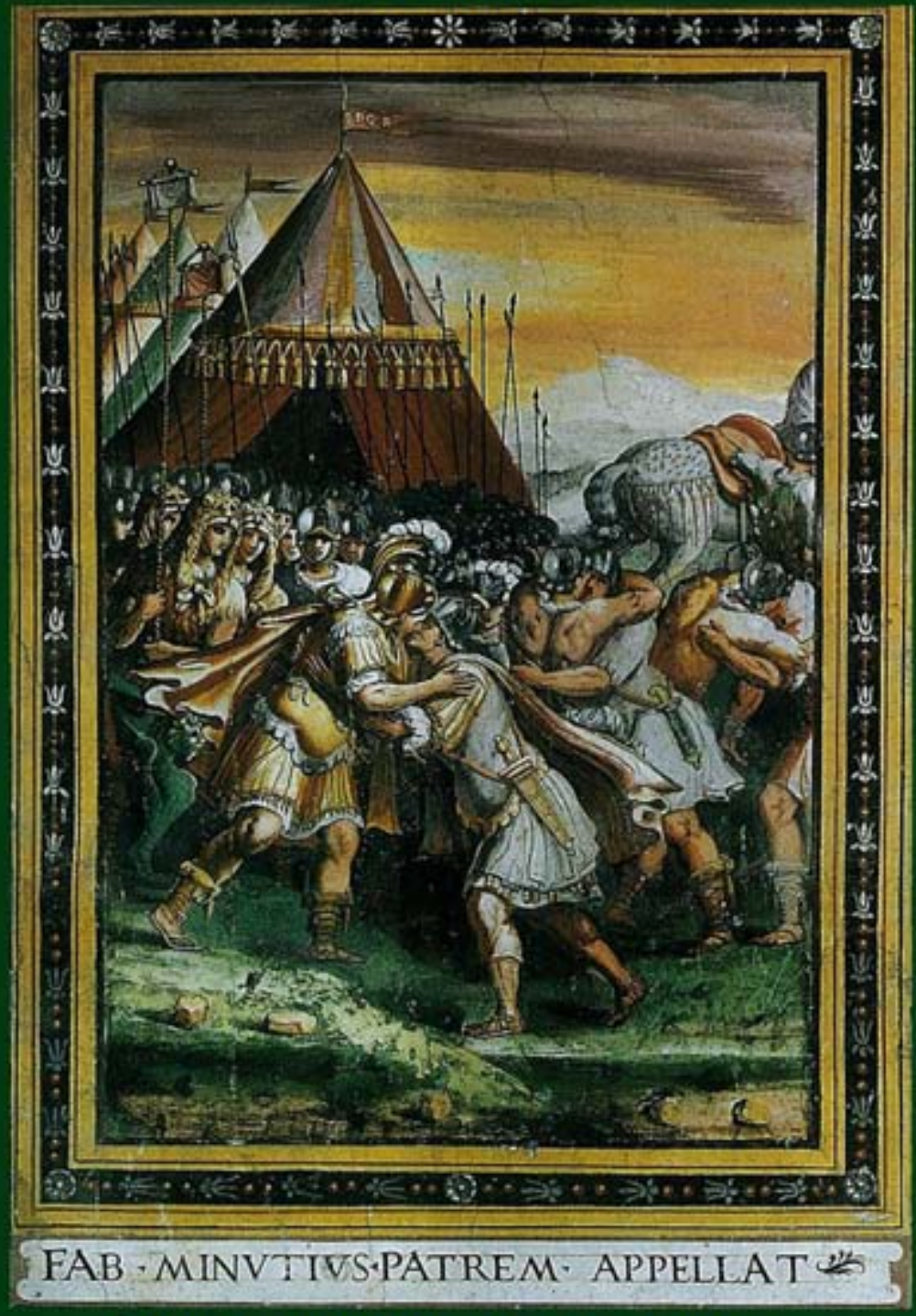

KATHOLIEKE UNIVERSITEIT LEUVEN IMPRENSA DA UNIVERSIDADE DE COIMBRA Leuven-Coimbra, 2008 
(Página deixada propositadamente em branco) 
José Ribeiro Ferreira, Luc van der Stockt \& Maria do Céu Fialho

EDITORS

\section{PhILOSOPHY IN Society}

\section{Virtues and Values in Plutarch}

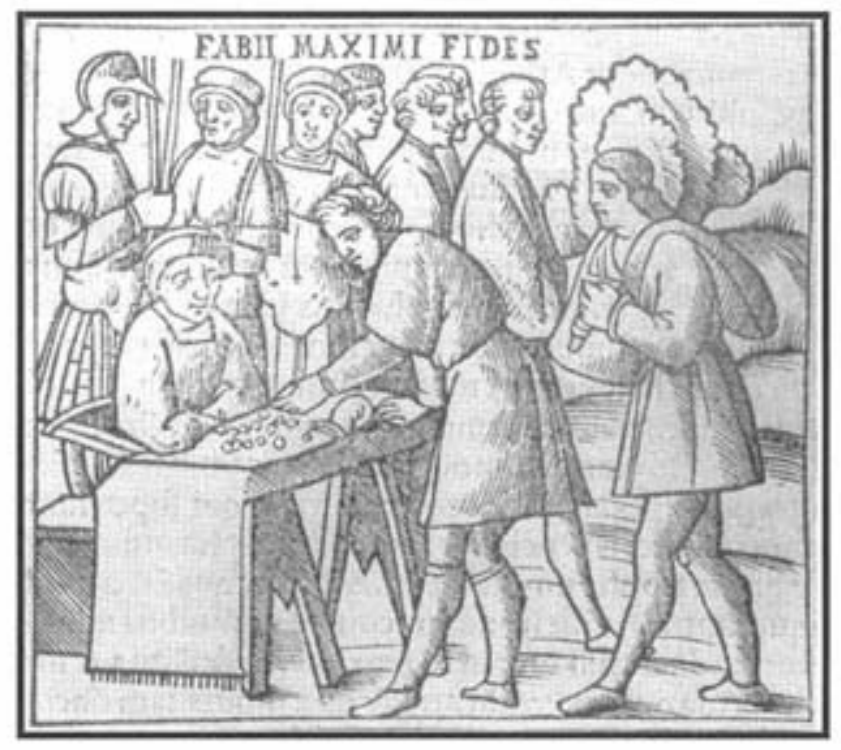

Fabius Maximus' Loyalty

Vitae Plutarchi Cheronei novissime post Jodocum Badium Ascensium longe diligentius repositae maioreque diligentia castigatae, cum copiosiore verioreque indice, nec non cum Aemilii Probi vitis, una cum figuris, suis locis apte dispositis, Venetiis 1516 , fol . 65v

\section{LEUVEN - CoIMBRA \\ 2008}

KATHOLIEKE UNIVERSITEIT LEUVEN

IMPRENSA DA UNIVERSIDADE DE COIMBRA 


\title{
First published 2008
}

\section{(C) Universiteit KATHOlieke Leuven \\ (c) Universidade Coimbra}

\author{
Published by \\ IMPRENSA DA UNIVERSIDADE COIMBRA \\ Imprensa da Universidade de Coimbra \\ Rua da Ilha, $\mathrm{n}^{\circ} 1$ \\ 3000-033 Coimbra (Portugal) \\ Email: imprensauc@ci.uc.pt \\ URL: http://www.uc.pt/imprensa_uc
}

ISBN: 972-989-8074-73-7

Legal Deposit: MA-140-2009

\author{
Printed in Spain by \\ IMAGRAF IMPRESORES, S.A. \\ c/ Nabucco 14 \\ 29006 Málaga \\ Tfno. 952328597
}

\section{Frontispiece:}

Fabius Maximus and Minucius (Francesco da Siena, Grottaferrata, Palazzo Abbaziale). We are grateful to the Archimandrita of the "Monastero Esarchico di Santa Maria di Grottaferrata", P. Emiliano Fabbricatore, for the authorization to reproduce this picture. 


\section{Éros, Arès et Aphrodite dans l'Érotikos Une reconsidération de la réponse à Pemptidès (ch. 13-18) \\ Françoise Frazier \\ UNIVERSITÉ DE PARIS X-NANTERRE}

Dans ce dialogue un peu à part de Plutarque que constitue l'Érotikos, dont le sujet, en plein accord avec le thème qui nous réunit, traite d'une question de morale pratique et s'ancre ainsi dans la " réalité quotidienne ", psychologique et éthique, là où les autres dialogues privilégient des questions physiques et métaphysiques, s'interrogeant sur le fonctionnement des oracles, la place de la lune dans le cosmos, la nature et la justice divine ${ }^{1}$, dans ce dialogue qui, formellement, entrelace une histoire d'amour particulière et une discussion, les images, qui suggèrent l'élan spirituel vers la Beauté véritable et divine, les exemples de tous ordres, qui, par leur caractère très souvent narratif, montrent l'amour inscrit dans la durée de l'existence humaine, bref, tout ce qui permet une « figuration » de la pensée, ancrant l'amour dans la réalité vécue tout en suggérant aussi sa valeur spirituelle, prend une importance particulière. J'ai déjà proposé plusieurs études autour de ce thème ${ }^{2}$ et je voudrais aujourd'hui poursuivre cet examen en m'attachant aux dieux traditionnels, qui

1 Ce qui lui valait d'être classé par K. ZIEGLER, « Ploutarchos of Chaironeia », RE XXI (1951), cols. 636-637, dans la catégorie des popularphilosophisch-ethischen Schriften, alors que les autres dialogues ressortissaient aux theologischen Schriften -à l'exception du De facie, rangé parmi les naturwissenschaftlichen Schriften, lequel est considéré par H. G. INGENKAMP, « Luciano e Plutarco: Due incontri con il divino ", Annali della facoltà di Lettere e Filosofia dell 'Università di Siena 6 (1985), p. 37, comme tout aussi théologique que les autres. L'Érotikos ne prétend pas à une réflexion théologique, mais il accorde néanmoins une attention aux choses religieuses qu'on ne trouve pas dans les traités éthiques et cette alliance est un autre aspect, non négligeable, de sa singularité.

2

Sur les images, voir Frazier, 1999 ; sur les exempla Frazier 2005 a.

José Ribeiro Ferreira, Luc Van der Stockt \& Marua do Céu Fialho (Edd,), Philosophy in Society

- Virtues and Values in Plutarch, Leuven-Coimbra, 2008, pp. 117-136. 
lient étroitement les deux dimensions existentielles de l'éthique et du religieux, et en privilégiant le premier aspect ${ }^{3}$.

Parmi eux, deux divinités majeures se détachent : Aphrodite, figure attendue, présente à la fois dans les passages périphériques consacrés au mariage ${ }^{4}$ et dans l'intervention centrale de Plutarque, aussi bien dans sa réponse à la question de Pemptidès sur la divinité d'Éros (ch.13-18) que dans le couronnement platonicien de cette réponse suscité par Soclaros (ch. 19-20), mais aussi Arès, qui n'est présent que dans la réponse à Pemptidès -à quoi il faut ajouter, dans un registre un peu différent, une utilisation récurrente de métaphores guerrières auxquelles il ne saurait être totalement étranger $^{5}$. Cette simple constatation inviterait déjà à centrer l'étude sur ces chapitres 13-18, mais il se trouve de surcroit que, lors d'une rencontre récente à Göttingen, le Professeur Görgemanns, étudiant ce qu'il considère comme un éloge d'Éros ${ }^{6}$, s'est attaché aussi à ces chapitres et a repris la lecture « rhétorique " proposée par D. A. Russell il y a déjà une dizaine d'années ${ }^{7}$ en l'appuyant sur un rapprochement avec les prescriptions d'Alexandre fils de Numénius pour la composition des éloges des dieux ${ }^{8}$.

Or la prééminence du modèle rhétorique ainsi établie me semble grosse de malentendus, pouvant déboucher sur une remise en question du genre même du texte, amenant à tout le moins à minorer indûment l'influence du Banquet sur ce passage, et, plus largement, à privilégier une certaine forme littéraire par rapport au fond l'explication ultime du passage étant un désir de l'auteur de se conformer à la personnalité juvénile du Plutarque ${ }^{9}$ : en jetant la lumière sur une éthopée d'ailleurs contestable, on rejette dans l'ombre l'apport du passage à l'ensemble de la réflexion sur l'amour et les liens qu'il entretient avec les autres parties du dialogue. Aussi me semble-t-il nécessaire de reprendre d'abord une vision d'ensemble des chapitres 13 à 18 qui propose une autre ligne de lecture et signale les rapprochements et les écarts avec les éloges du Banquet de Platon. De fait, il ne s'agit pas de nier que ce texte ait des accents encomiastiques, mais de montrer et ce qu'ils doivent à Platon et surtout ce qu'en fait Plutarque. L'évocation de la puissance d'Éros est à l'évidence un

J'ai étudiê l'aspect religieux in FrAzıER, 2005b.

Voir Daphnće en 5. 751 E [citation de Solon] et surtout 752 B et Plutarque en 23. 768 E - 769 A.

Voir VALVERDE, 2004/2005.

Cette dimension est indéniable, mais l'analyse de Gotrel.AND-OudoT, 2006, p. 76, qui associe "Apologie et éloge du dieu Amour ", me paraît plus exacte et lorsque j'ai moi-même évoqué un éloge du Dieu (FrazıER, 2005/2006), j'entendais mettre en lumière une tonalité valable pour l'ensemble du dialogue et non pas seulement la structure formelle d'un passage particulier.

RUSSELL, 1997 ,

GörgemanNs, 2005, qui cite et traduit le texte d'Alexandre p. 193-195.

Gorgemanns, 2006, p. 9, 26 et en part. $29: \approx$ Die Nähe zur Rhetorik fügt sich auch in das Charakterbild des jugendlichen Plutarch ein ». 
thème d'éloge, mais pourquoi choisit-il, pour l'exalter, de le comparer à Arès et à Aphrodite et que faut-il en particulier penser de l'introduction de ce passage (759 E310), qui n'a pas encore été suffisamment élucidée ? L'étudier, c'est mettre en lumière la dimension éthique et psychologique de la réflexion, dominante dans la comparaison entre Arès et Éros, tandis que la relation est plus complexe avec Aphrodite et permet d'associer considérations éthiques et dimension cosmique.

\section{Forme et contenu de la réponse à Pemptidès}

S'il n'est pas faux de lire les chapitres 13 à $18^{10}$ comme un éloge, une telle lecture ne prend toutefois pas suffisamment en compte le mouvement du texte, son origine comme son développement. De fait, avant de prendre la forme d'un éloge ${ }^{11}$, cette longue intervention de Plutarque est d'abord suscitée par une question de Pemptidès, qui, explicitement, déplace le débat d'un problème particulier à un plan général, du mariage d'Isménodore et de Bacchon à la nature de l'amour ${ }^{12}$-question d'ailleurs préparée par le premier débat contradictoire sur la valeur respective de l'amour des garçons et de l'amour conjugal, oủ était déjà en jeu « ce qui mérite vraiment le nom d'Amour " et qui prend donc forme dans la bouche de l'austère Pemptidès : « J'aimerais entendre de vous ce qu'avaient en vue pour déclarer Éros dieu les promoteurs de cette opinion " $(12,756 \mathrm{~A})$. Une telle question ressemble fort à celles qu'on pouvait poser au professeur-conférencier de philosophie et elle contribue à asseoir la figure dominante de Plutarque, qui s'affirmera encore lorsqu'il sera pressé au chapitre 19 de développer l'accord avec la religion égyptienne par Soclaros. Sans doute est-ce là un anachronisme, mais outre qu'il n'a rien de très surprenant dans la tradition platonicienne, où l'on n'a que faire de la vraisemblance matérielle, cette superposition des figures, du jeune marié amoureux et du vieux philosophe heureux en ménage, père du narrateur, loin d'en constituer une faiblesse, l'enrichit et ajoute à l'entrelacement de l'histoire et du dialogue un miroitement des temps qui fait de Plutarque la meilleure illustration de la théorie qu'il développe ${ }^{13}$.

Sans doute est-on de la sorte très loin du questionnement socratique, mais on ne l'est pas moins des éloges monologiques des cinq premiers intervenants du Banquet. Si l'on retrouve le ton poli qui est celui de la société amicale de Plutarque et qu'il évoque si bien dans les Propos de Table, son intervention n'en a pas moins une certaine saveur " diatribique ", avec un Plutarque qui se tourne vers ses inter-

Et peut-être 13 à 20 , si l'on voit dans le développement platonicien l'exaltation du bienfait suprême d'Éros et done le couronnement de l'éloge.

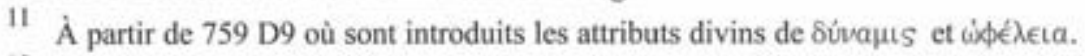

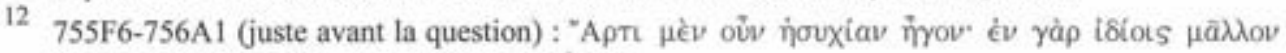

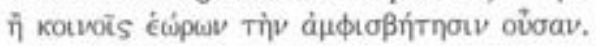

13

La très belle analyse de Sirınel.ı, 2000, p. 412, me semble à cet égard décisive. 
locuteurs, Pemptidès d'abord, mais aussi Daphnée et Zeuxippe, pour solliciter leur avis, leur approbation, leur intervention, au cours des trois parties qu'il détache luimême par des articulations très nettes ${ }^{14}$. Je prendrai pour exemple la première, la plus complexe, qui mêle apologie, réfutation et éloge pour établir la divinité d'Éros (13-16. 759 D), et dont les affinités avec les prescriptions d'Alexandre ne me semblent pas le trait le plus frappant, ni surtout le plus éclairant. D'abord, indépendamment de l'origine, rhétorique ou non, de ses composantes, l'exposé se déroule en établissant avec les autres assistants une certaine forme de dialogue, ou de prise à partie, qui n'est pas sans évoquer les entretiens philosophiques contemporains ; ensuite elle présente un certain nombre de points communs avec d'autres œuvres de Plutarque : son mouvement, qui conduit d'une position apologétique, où est défendue la divinité d'Éros et souligné le danger de sa mise en cause, à une exaltation de cette divinité qui patronne l'union amoureuse, se retrouve dans la dernière partie, à propos du mariage, sans doute attaqué par Zeuxippe ${ }^{15}$, et anime aussi le Sur les oracles de la Pythie ${ }^{16}$; son contenu et les réflexions sur la nature et la fonction du dieu peuvent être rapprochés de thèmes du Sur Isis et Osiris ${ }^{17}$. Enfin, outre ces échos internes à l'œuvre de Plutarque, la ressemblance avec les éloges du Banquet est indéniable ${ }^{18}$ et cette première partie peut se lire comme une adaptation de la première partie que consacrent, Phèdre à la genesis du dieu (178 a sq) et Agathon à sa nature (195 a), point qui est aussi le premier traité par Socrate et Diotime et leur permet d'établir qu'Éros est un daimôn et non pas un dieu (201 e sq). Sans reprendre le procès en infidélité fait à Plutarque sur ce point par des générations de critiques, il faut souligner plutôt comment l'alternative qu'il choisit, non pas theos ou daimôn, qui met en lumière l'intentionnalité de l'amour et en fait la meilleure métaphore du désir philosophique, mais theos ou pathos, situe l'amour hic et nunc, et constitue peut-être une certaine manière, la sienne, de penser le metaxu, en se focalisant sur la dimension vécue, éthique et religieuse à la fois, où une puissance divine patron-

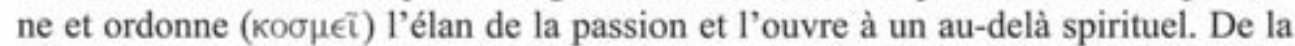
même manière, l'éloge en forme qui suit révèle un contenu sans doute plus riche et

Voir en appendice l'analyse de la réponse à Pemptidès, qui dégage à la fois la progression argumentative et la forme dialogique adoptée.

15

Les sous-titres choisis dans son édition par Flacel.térE, 1980 sont assez suggestifs : * Apologie de l'amour conjugal " pour le chapitre 21 (p. 95), puis « Grandeur du mariage » pour le chapitre 23 (p. 100).

C'est du moins ce que j'ai essayé de montrer dans une communication toujours à paraitre, faite au colloque Interpreting Composition in Phutarchos (Louvain, 5-7 juillet 2001), « Delphes dans tout son éclat. Le De Pythiae oraculis couronnement des Dialogues Pythiques $\$$.

17 J'espère traiter ailleurs ce sujet.

18 Voir VALVERDE, 2002, n.116, p. 80 : il ajoute à la référence à Agathon et au Banquet la disposition adoptée par Socrate in Phaedr: 237 c-d. 
significatif si on le situe d'abord dans la pensée de son auteur avant de se référer à un modèle rhétorique et à un répertoire de thèmes.

La divinité d'Éros affirmée, Plutarque choisit de développer deux attributs

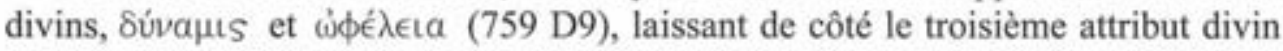

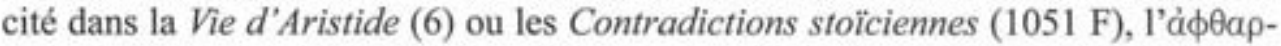
бía, l'incorruptibilité, qui accuse l'écart ontologique entre divin et humain et n'a pas sa place ici $^{19}$. Les bienfaits divins correspondent sans doute à un thème d'éloge, développé par Phèdre, Agathon et Diotime ${ }^{20}$, mais aussi à une conviction intime de Plutarque, qui a animé toute l'argumentation de la première partie fondée sur le patronage divin de toutes nos activités ${ }^{21}$ et qui couronne ainsi l'ensemble de son intervention. Entre les deux, il s'attarde sur la puissance du dieu, autre thème d'éloge assurément, que traitent Éryximaque, Aristophane et Diotime dans le Banquet $^{22}$ : il choisit de la traiter sous la forme d'une syncrisis, procédé rhétorique, dont les Vies parallèles montrent assez qu'il lui est cher, mais qui n'est pas absent non plus de la partie de l'éloge d'Agathon consacrée à l'arétè du dieu (196 b-197 b), où, comme chez Plutarque, la puissance se fait prévalence sur toute autre. Thème encomiastique bien à sa place à ce moment du texte, il est cependant loin de se limiter à ce passage : c'est au contraire un thème qui parcourt tout le texte ${ }^{23}$, qui se manifeste dans l'histoire même, par l'inspiration qui s'est emparée de la sage

19 Plutarque ne reprend pas dans cette partie la forme d'immortalité que peut conférer l'amour ; c'est Daphnée qui a évoqué le flambeau des générations en $752 \mathrm{~A}$, et Plutarque reprendra fugitivement le thème en $770 \mathrm{~A}-\mathrm{B}$, au niveau cosmique (passage commenté infra) ; le mouvement vers l'Être et le retour à la plaine de la vérité ne sont pas explicitement mis en relation avec l'immortalité, mais inscrits dans le cycle des renaissances (766 B).

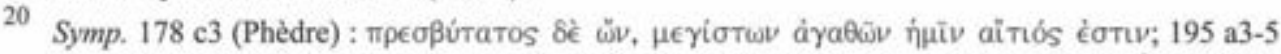

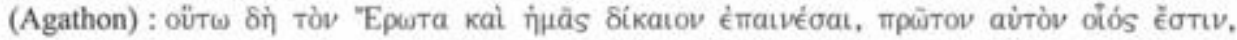

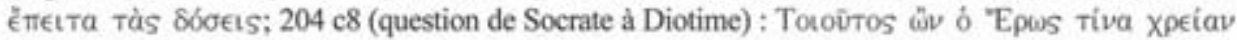
ËX

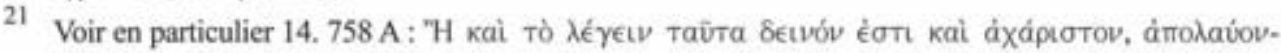

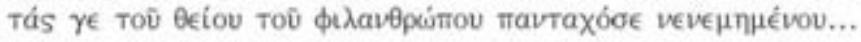

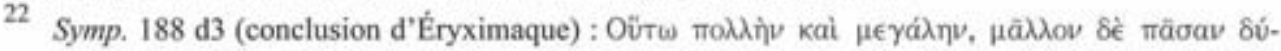

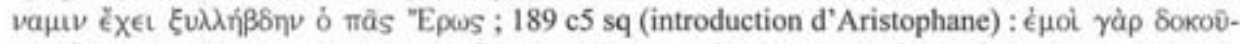

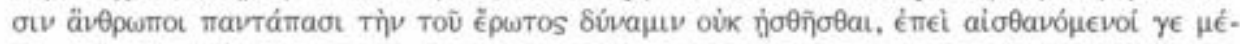

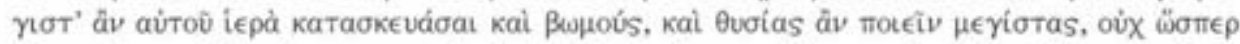

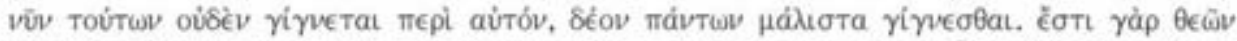

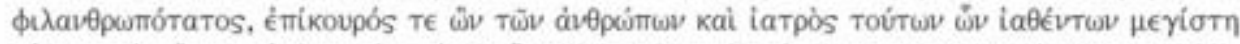

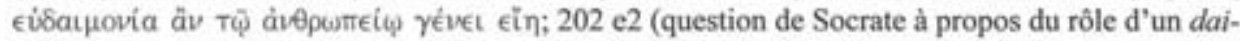

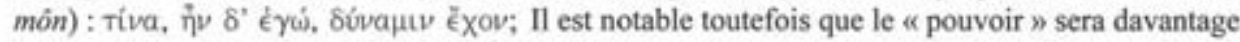
souligné dans l'éloge d'Alcibiade, comme puissance de Socrate, qui est aussi ascendant de l'amour philosophe ( $216 \mathrm{c} 8$ et $218 \mathrm{e} 2)$.

Voir FrAziER, 2005/2006, 95-99. 
Isménodore ${ }^{24}$, et a été repris et amplifié par l'introduction de la théorie des maniai, parmi lesquelles la passion amoureuse a été désignée comme la plus forte ${ }^{25}$; cette prise de possession, source de tous les héroĩsmes, s'épanouira dans l'ultime réponse à Zeuxippe, où sera exaltée la résistance à toute coercition de «ceux qui ont installé Éros comme maître dans leur âme $\mathrm{e}^{26}$ ". Cette dimension psychologique prédomine aussi dans l'introduction de la partie consacrée à l'exaltation de la puissance d'Éros, qui est précisément le passage très délicat où l'on trouve ensemble Arès et Aphrodite.

\section{Arès et Aphrodite moins puissants qu'Éros (759 D9-E10)}

Cependant puisque nous $<$ distinguons $>$ les dieux surtout par la puissance et l'utilité, de même que parmi les biens humains, ce sont la royauté et la vertu que nous pensons et disons les plus divins, il est temps de considérer d'abord si Éros le cède en puissance à quelque autre divinité. Or

Grand est le pouvoir qui à Cypris assure la victoire

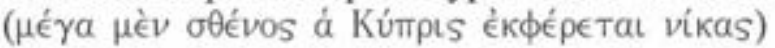

comme le dit Sophocle (Trach. 497), grande aussi la force d'Arès ( $\mu \epsilon \gamma a \dot{\lambda} \eta \eta \delta^{\prime}$ $\dot{\eta}$ Toũ "A $\rho \in O S ~ i \sigma \chi u ́ s)$, et, d'une certaine manière, nous voyons que la puissance de tous les autres dieux est répartie entre eux deux : la puissance d'appropriation du beau de l'une et la puissance de résistance au laid de l'autre sont inscrites dès l'origine dans les âmes, comme Platon quelque part ... les formes

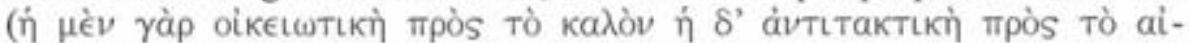

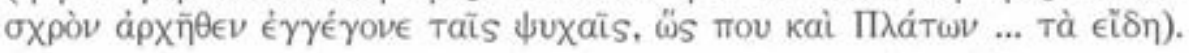

Le texte est ouvertement présenté comme platonicien, mais la référence est tronquée et le complément proposé par les savants modernes, qui renvoie à la distinction des parties de l'âme ${ }^{2}$, ne permet d'établir de correspondance parfaite avec aucun texte platonicien conservé. Deux propositions ont été faites, dont les promoteurs reconnaissent eux-mêmes les imperfections : R. Flacelière met en avant le mythe de l'attelage ailé (Phèdre 246 b) et identifie les deux tendances aux deux chevaux, c'est-à-dire à l'epithymia et au thymoeidés ${ }^{28}$, mais le désir incarné par Aphrodite dans notre dialogue n'a rien de négatif; A. Barigazzi préfère se reporter à la division des parties de l'âme de la République (IV 439 e), mais si le thymoei-

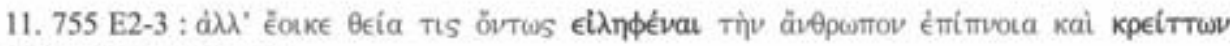

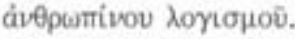

16. 759 A-B établit que toutes les autres passions s'apaisent et leur oppose trì $\delta^{\prime}$ É

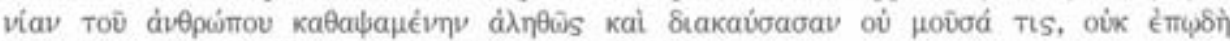

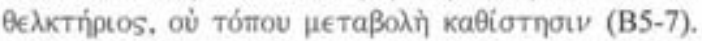

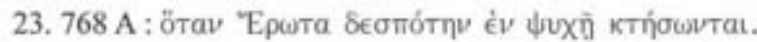

FLACELIERE, 1980 comme GÖRgEMANNS, 2006 adoptent la conjecture de Wyttenbach $<\delta t \epsilon i \lambda \epsilon$ Tīs

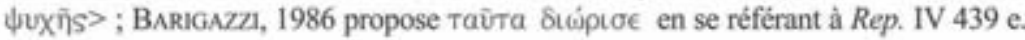

Flaceliére, 1980, p. 142 (= n. 4 de la p. 76). 
dés, bien orienté, correspond toujours, sans grande difficulté, à Arès, il faudrait identifier Aphrodite avec le voũ ${ }^{29}$, ce qui ne laisse pas de surprendre. M. Valverde pour sa part renvoie aux deux textes, mais en s'arrêtant davantage sur le Phèdre et en signalant, sans explication, que les deux tendances ici sont bonnes ${ }^{30}$, tandis que H. Görgemanns ne cite que la République ; alors que, dans une première version

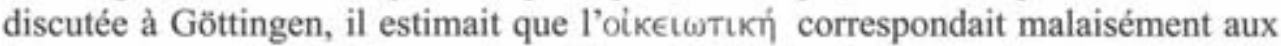
deux autres parties de l'âme, rationnelle ou désirante, il s'est finalement rallié à l'i-

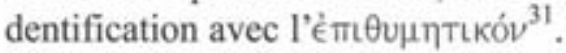

La source platonicienne parait donc difficile à saisir, mais avant de chercher (ou de renoncer) à la déterminer, avant même de conclure, avec $\mathrm{R}$. Flacelière, que " Plutarque veut dire, je crois, qu'en limitant l'examen à Aphrodite et Arès, on aura, en réalité, épuisé la question relative à la comparaison de la puissance d'Éros avec celle de tous les autres dieux ${ }^{32}$ ", il faut essayer de déterminer plus précisément la portée éthique et psychologique de cette division, et, pour ce faire, considérer de plus près le vocabulaire employé pour désigner ces deux grandes puissances. Les deux adjectifs mettent en valeur des mouvements opposés et complémentaires,

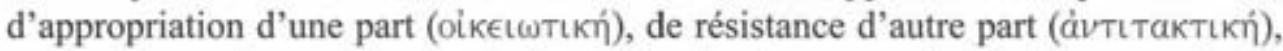
qui transposent en quelque sorte dans l'âme les deux grands principes cosmiques, philia et neikos', qui animent tout l'univers ; du caractère moral de cette transposi-

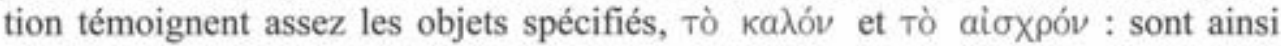
mises en relief deux tendances dynamiques de l'âme, qui peut-être disent à la manière platonicienne le " système binaire, attraction-répulsion » sur lequel les Stoïciens construisaient leur propre système éthique ${ }^{33}$, et le disent dans des termes suffisamment rares pour mériter eux aussi l'attention.

La tendance la plus facile à cerner, la force d'opposition et de résistance propre à Arès, est en effet désignée par un hapax, l'adjectif ảvTLTaKтLKท́, dont on peut rapprocher l'action défensive et bénéfique attribuée à Arès dans les Daidala (frg 157

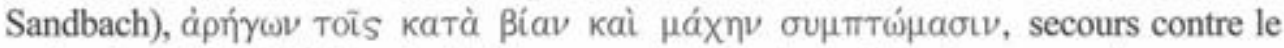
mal qui se déploie là dans la cité alors que notre texte se situe dans une optique morale : de façon plus spécifique, l'action d'Arès a été définie un peu plus haut, au début du

GORgemanns, 2006, p. 156, n. 203, qui renvoie à OpSOMER, 2006, p. 229, lequel rapproche ce dèsir du Beau du désir attribué à Isis, identifiée à la matière, in De Iside 53, 372 E-F (ËX€t $\delta \dot{~} \sigma u ́ \mu \phi u$ -

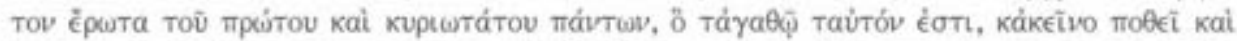

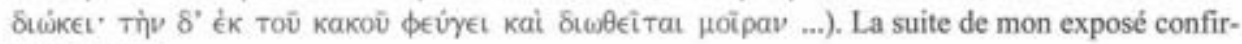
me ce choix.

32

33

BARIGAZZI, 1986, p. 115.

VALVERDE, 2003, p. 80 , n. 118.

Flaceliêre, 1980, p. $142(=$ n. 3 de la p. 76$)$.

Je reprends les termes de C. Lévy, Les philosophies hellénistiques, Paris, 1997, p. 160. 


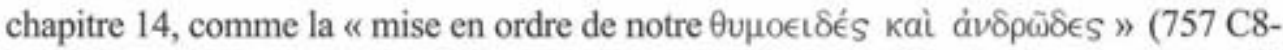
9). L'interprétation est plus délicate pour l'action d'Aphrodite, mais, pour rare qu'il soit, l'adjectif n'est pas un hapax et il existe un autre texte où l'on trouve ainsi désignée une tendance de l'âme, le seul, à ma connaissance. Il s'agit, dans le Didaskalikos d'Alcinoos, c'est-à-dire dans un texte médioplatonicien, d'un développement situé à l'intérieur de la partie morale de l'exposé et consacré à l'immortalité de l'âme ${ }^{34}$ :

L'âme des dieux possède elle aussi le discernement (Tò крıтเкóv) que l'on pourrait également appeler faculté de connaître, ainsi que la faculté impulsive (тò ó $\rho \mu \eta\rceil \leftarrow к o ́ v)$, c'est-à-dire capable d'excitation, et la faculté d'appropriation (тò oíкŁL humaines, mais du fait de leur venue dans le corps, elles subissent un certain changement : à la faculté d'appropriation correspond la partie concupiscible de l'âme, à la faculté impulsive, la partie irascible (ớo $\mu \in T a \beta 0 \lambda \eta ̀ ̀ \nu ~ \lambda a \mu \beta a ́-$

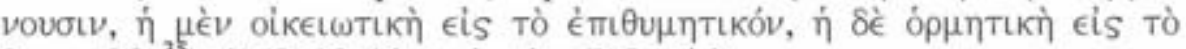

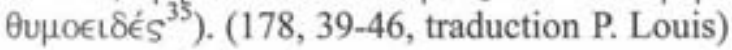

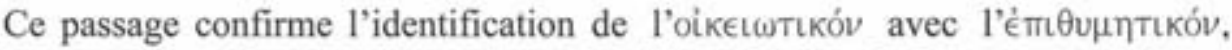
mais surtout il se situe au même niveau que l'introduction de Plutarque, au niveau divin, et il n'a pas moins intrigué les spécialistes du médioplatonisme ${ }^{36}$ que notre passage n'a déconcerté ses éditeurs. J. Dillon, commentant le texte d'Alcinoos, a suggéré d'y voir l'influence du Phèdre et des chariots divins, qui aurait amené les platoniciens à concevoir qu'il « devait y avoir dans les âmes divines des archétypes équivalents aux parties irascible et concupiscible de l'âme humaine $\mathrm{e}^{37}$ " ; chaque cheval étant évidemment noble lorsqu'il est attelé à un chariot divin, on comprend aisément pourquoi, à ce niveau, les deux tendances sont pleinement positives, chez Plutarque comme chez Alcinoos ${ }^{38}$.

Ces tendances concernent plus précisément la partie passionnelle de l'âme, dont le De la vertu éthique souligne l'importance dans la vie morale : sans elle, la raison serait inerte, et l'on trouve à la fin de ce traité un passage où Plutarque distingue nettement

34

35

36

37

38

Cet argument soulève implicitement le problème, débattu, de la mortalité ou de l'immortalité de la partie irrationnelle de l'âme (voir infra n. 36).

WhITTAKER, 1990, n. 413, relève que c'est le seul passage où Alcinoos emploie le terme platonicien, alors que partout ailleurs il préfêre l'aristotélicien $\theta u \mu$ uóv.

K. Alt, Gott, Götter und Seele bei Alkinoos, Stuttgart, 1996, p. 32, n. 125, ne pense pas cette théorie des âmes divines propre à Alcinoos, au contraire de DıLoN, 1995, p.160 ; la perplexité vient en particulier de ce que l'affirmation d'une préexistence de la partie irrationnelle de l'âme ne s'accorde pas avec la mortalité que semble lui attribuer Alcinoos.

DILLON, 1977, p. 292.

WHITTAKER, 1990, p. 132, n. 410, signale que Dillon n'a pas d'autre témoin de cette théorie qu'Alcinoos : peut-être le texte de l'Érotikos peut-il être considéré comme un second témoignage. 
l'excès de passion, à éviter, de la passion elle-même, dont les mouvements sont à la fois consubstantiels à l'âme incarnée, et indispensables à son activité. C'est ce qu'ont compris les législateurs, qui encouragent l'émulation dans le domaine politique et au combat font jouer des airs excitants ; ce qu'ont compris aussi les éducateurs, Stoïciens compris, qui manient l'éloge et le blâme et Plutarque conclut :

Ainsi un pédagogue laconien n'avait pas tort, quand il disait qu'il voulait amener l'enfant à jouir des bonnes actions et à souffrir des mauvaises (Toîs

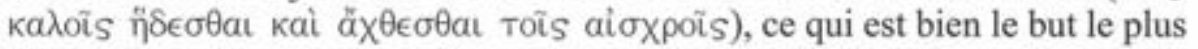
élevé et le plus beau que l'on puisse assigner à l'éducation, telle qu'elle convient à un être libre. (452 D6-9)

Ce dernier exemple, pédagogique, montre en quelque sorte l'actualisation des

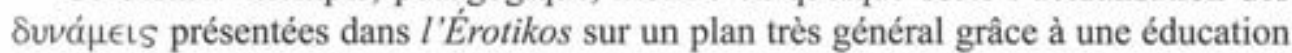

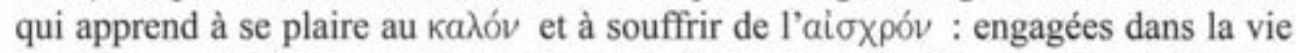
pratique, ces tendances prennent ainsi une dimension affective et suscitent des sentiments moraux de plaisir et de déplaisir, mais elles se concrétisent aussi en vertus. Le traité insiste sur la justice et la sagesse ${ }^{39}$, tandis que l'Érotikos sélectionne des situations où les mouvements d'appropriation et de répulsion rattachés à Aphrodite et a Arès sont replacés dans les domaines traditionnels des deux divinités, l'amour et la guerre. Et l'on se retrouve ainsi aussi dans le monde des passions, où dominent epithymia et hédonè avec la première, thymos avec le second. Par rapport à la « mise en perspective psychique " initiale, très générale et originale, le développement prend un caractère plus convenu qu'on peut regretter, mais s'il réduit le champ d'application des tendances, il ne réduit pas l'intérêt de l'introduction, et n'en perd pas pour autant lui-même tout intérêt.

\section{Éros et Arès}

Convenue, la comparaison avec Arès, qui fait d'Éros la source du plus grand des courages, prend cependant un peu plus de relief si on la replace dans l'ensemble du texte et si, d'abord, l'on revient au passage du De la vertu éthique déjà évoqué ${ }^{40}$; après y avoir rappelé l'utilisation des instruments de musique pour galvaniser les courages, Plutarque continue :

Car ce n'est pas seulement en poésie que, comme le dit Platon, le possédé des Muses et l'inspiré rendent ridicule le professionnel rompu à son

39

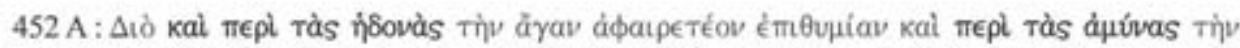

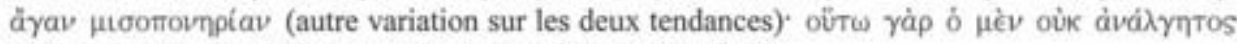

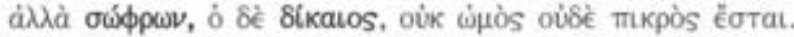

La comparaison avec le raisonnement spécieux d'Agathon (Symp. 196 d) est aussi à son avantage :

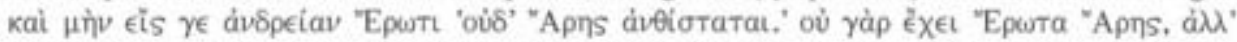

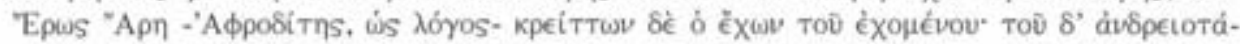

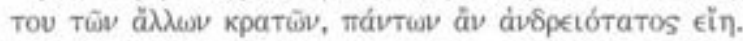


métier, mais aussi bien dans les batailles, où l'élan passionnel et la force

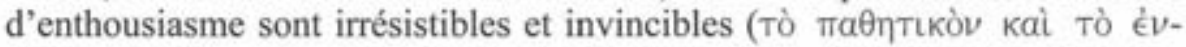

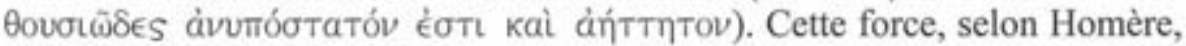
les dieux la suscitent en l'homme :

Il dit et au pasteur d'hommes il insuffle une grande fougue (O 262) et encore

Ce n'est pas sans l'aide d'un dieu qu'il montre ici telle fureur (E 185), comme si la passion qu'ils adjoignent ainsi au raisonnement était pour celui-

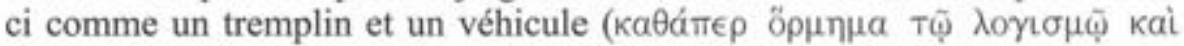

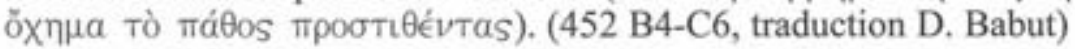

Cette soudaine référence au Phèdre et à l'inspiration poétique a semblé mal raccordée à D. Babut, voire saugrenue $\mathrm{e}^{41}$; son insertion donne cependant à penser que ce thème psychologique importe à Plutarque et sa récurrence dans l'Érotikos semble le confirmer ; en outre, le passage du De la vertu peut éclairer une particularité de l'exposé sur les maniai de notre dialogue que les savants se contentent en général de noter sans la commenter : l'adjonction à la liste platonicienne de la mania guerrière $(16.758 \mathrm{~F})$, la première dont il est noté ensuite, à travers une citation d'Homère (Z 121-122), qu'elle s'éteint dès que le guerrier quitte les armes, alors que rien n'apaise l'amour (16.759 A), de même qu'elle a été la dernière citée avant l'introduction de la mania amoureuse.

Ce rapprochement entre les deux domaines et les deux divinités, présent dès le début de la réponse à Pemptidès, où Arès, « ce dieu qui, comme sur un planisphère de bronze, est aux antipodes d'Éros ", était victime aussi des pires injures (757 AB) avant que son patronage du thymoeidés et des combats ne soit avancé comme indice de l'existence d'un dieu symétrique pour patronner le philètikon (757 C-D), attire l'attention sur de puissants mouvements de l'âme et culmine dans la syncrisis de la puissance d'Arès et d'Éros, où elle prend une tonalité héroïque, préparée peutêtre justement par l'introduction de la mania martiale. Plutarque en tout cas peut puiser à pleines mains dans la tradition épique et rejoint sur bien des points l'éloge de Phèdre dans le Banquet ${ }^{42}$ pour affirmer la supériorité d'Éros. Il substitue ainsi à l'Éros délicat des poètes un Éros courageux $(760 \mathrm{D})$, pour finalement le substituer

BАвUт, 1969, p. 41: « Mais ce qui frappe surtout, c'est que ces matériaux qui, en définitive, constituent l'essentiel du chapitre, ne se prêtent pas toujours à l'exploitation que veut en faire l'auteur. C'est le cas du mot de Diogène sur Platon (452 D) .... On en dira autant de l'allusion à la théorie platonicienne de l'inspiration poétique (452 B), qui paraît presque saugrenue dans un tel contexte. $n$ Amat. $760 \mathrm{D}$, ardeur à défendre son aimé $=S y m p, 179 \mathrm{a} ; 760 \mathrm{E}$, ardeur au combat devant son aimé = 179 a ; 761 B institution chalcidienne, panoplie et bataillon thébains // 178 e (institution d'une armée d'aimés) ; peuples et héros d'oủ Alceste (et Orphée) $761 \mathrm{E}-\mathrm{F}=179 \mathrm{~b}$-d. 
à Arès lui-même $e^{43}$ : il est la plus grande source de courage et l'exemple ultime, cité aussi par Phèdre, en est Alceste, une femme, " qui n'a rien de commun avec Arès " (761 E), et dont le sacrifice suscite une nouvelle confrontation, où Éros, plus puissant qu'Arès, se mesure désormais à Hadès et l'emporte sur lui aussi ${ }^{44}$. Affleure ainsi le thème de l'amour plus fort que la mort, qui s'épanouira dans la dernière partie du dialogue avec les exemples de Camma et d'Empona, plus fort parce que, à l'instar de ces femmes, il ne la craint pas, plus fort aussi —et c'est ce que montrent les chapitres 19-20 platoniciens - parce qu'il ramène l'âme à l'Être.

Peut-être enfin faut-il ajouter aux « victoires » d'Éros le mariage d'Isménodore et de Bacchon et l'on aurait alors une des explications possibles à l'emploi insistant de la métaphore guerrière dans l'histoire : non seulement elle met l'accent sur le caractère passionnel inhérent à l'amour, mais l'apaisement final de la " guerre ${ }^{45}$ " et le triomphe de l'amour inscrivent aussi dans le récit la supériorité d'Éros sur Arès soutenue dans la discussion. Sur le plan psychologique en tout cas, celui sur lequel se situe l'introduction à l'éloge de la puissance d'Éros, la mise en parallèle avec Arès assoit la conception de l'amour comme force motrice de la vie morale et sa puissance d'élévation sera confirmée dans la partie sur les bienfaits, où Plutarque met l'accent sur la métamorphose morale, qui donne au lâche de la bravoure, au ladre de la générosité (762 B), qui remplit l'âme basse de noblesse et d'honneur (762 E), thème repris encore dans la dernière partie avec Laĩs (ch. 21), la courtisane qu'Éros rend sôphrôn alors qu'elle n'était jusqu'alors vouée qu'aux aphrodisia.

\section{Éros et Aphrodite}

Cette dimension éthique, qui associe Aphrodite et les aphrodisia, prédomine dans le passage consacré à la comparaison des deux puissances d'Éros et d'Aphrodite —et l'on est assez loin alors de l'oikєı exergue du passage. Son mouvement même a souvent déconcerté les commentateurs, qui y ont vu une critique de l'amour des femmes. La suite des arguments est pourtant assez claire, qui, de même que l'exemple d'Alceste montre ce que peut faire Éros sans Arès, s'applique à montrer le peu de prix d'unions où Aphrodite est privée d'Éros, les maris étant alors prêts à prostituer leur femme par ambition, et l'attachement sacré que suscite au contraire Éros - le dévouement des érastes est alors la meilleure preuve puisque justement Aphrodite n'a pas de part dans leurs relations ; ce caractère sacré est enfin reconnu par Alexandre lui-même, qui s'abstient des aphrodisia avec des femmes qu'aiment ses compagnons, sacrifice qui est

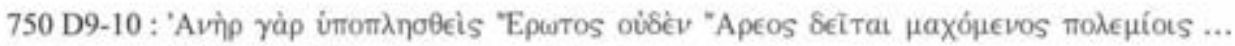

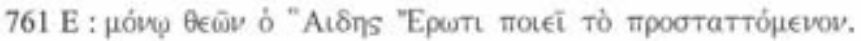


un hommage du plus puissant des rois à la puissance supérieure d'Éros ${ }^{46}$. Si ce passage est plus délicat à suivre, c'est aussi qu'il est plus original et qu'on n'en trouverait aucun écho dans aucun des éloges du Banquet; il contredit même quelque peu l'affirmation initiale de Daphnée, proclamant, pour s'opposer à Protogène, défenseur de la vision traditionnelle d'un amour des beaux garçons sans aphrodi-

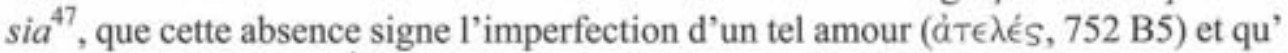
« il ne peut y avoir d'Éros sans Aphrodite, elle qu'il a reçu pour lot des dieux d'assister et d'escorter ". À la théorie classique de l'amour philosophique, Daphnée répond par la vision non moins traditionnelle d'Éros desservant d'Aphrodite, vision que conserve Platon lui-même lorsqu'il invente le mythe de la naissance d'Éros, expliquant «pourquoi Amour est le suivant d'Aphrodite et son desservant : parce qu'il a été engendré pendant la fête de naissance de celle-ci et qu'en même temps l'objet dont il est par nature épris, c'est la beauté, et qu'Aphrodite est belle ${ }^{48}$ ". Aphrodite reste première chronologiquement, mais aussi ontologiquement, comme objet d'amour, c'est-à-dire comme ce dont l'Amour est manque, parce qu'elle est liée à la beauté.

Or Plutarque, lui, l'associe au plaisir et à l'union charnelle et, dans la réponse à Pemptidès, s'attache à renverser la hiérarchie traditionnelle. Si, montrant que l'attaque contre Éros met en danger tous les autres dieux, il cite, immédiatement après Zeus, la puissante Aphrodite (756 D), célébrée par Empédocle et Sophocle, il commence cependant aussitôt à inverser leur relation par une paronomase, rappelant que son cuvre de fécondité, ergon grand et admirable, n'est pourtant que parergon lorsqu'Éros est présent, œuvre secondaire, simple " à-côté ». Éros apporte quelque chose de plus et permet à l'amour de se distinguer d'un simple appétit —ce à quoi le réduisait au contraire Protogène ; et Aphrodite, qui reste encore sujet de la phrase, peut écarter la simple satiété du plaisir et établir des liens durables et intimes,

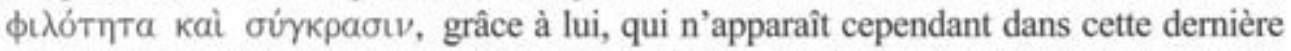
phrase que sous la forme discrète d'un datif instrumental, c'est-à-dire dans un rôle d'adjuvant ${ }^{49}$. Absence déjà révélatrice, la mania amoureuse est mise ensuite sous le

L'étonnement de RusselL, 1997, p. 106, devant le retour d'exemples féminins n'a ainsi pas lieu d'être, puisque la comparaison n'est plus entre hommes et femmes, mais entre Éros et Aphrodite, sentiment amoureux et commerce amoureux ; seule la présence du premier rend la relation et les aimés (ou aimées) inviolables et sacrés, indépendamment du sexe ; le retour final à des exemples féminins, loin d'être étonnant, confirme qu'il n'est pas ici question de distinguer les sexes. Dans le même esprit, il dénie aussi la qualité d'erotikos à qui ne vise que les aphrodisia avec une épouse désagréable (750 E), et, sur ce point, l'argumentation que je viens d'analyser lui donne raison.

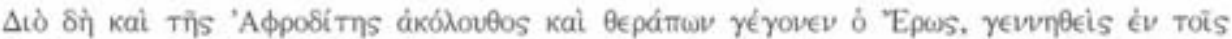

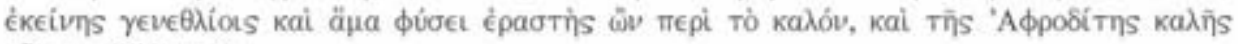
oǔonฺ. (203 $\mathrm{cl}-4)$.

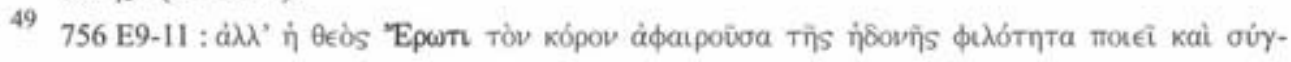
крабเv. 
patronage du seul Éros $\left(759 \mathrm{~A}^{50}\right)$, là où Platon la confiait à Éros et Aphrodite (Phoedr: 265 b), mais c'est dans la comparaison des puissances que l'inversion s'affirme clairement, avec un retournement frappant de l'affirmation de Daphnée : il n'est plus question d'un Éros qui n'est pas le vrai sans Aphrodite, mais d'une Aphrodite dont l'œuvre s'achète une drachme si Éros n'est pas à ses côtés ${ }^{51}$, et la même subordination se retrouve dans le hieros logos suscité par Soclaros, où Aphrodite est assimilée à la Lune, " par elle-même dénuée de force et pleine d'obscurité, quand le soleil ne vient pas l'illuminer, comme Aphrodite quand Éros n'est pas à ses côtés » (764 D7-9).

Cette inversion n'implique nullement un rejet des aphrodisia et Plutarque, s'il semble contredire Daphnée, voire se contredire lui-même, qui, dans sa polémique contre les Stoïciens, reproche à ses adversaires de ne pas « réserver le nom d'éros à ce que tous et toutes conçoivent sous ce nom et désignent ainsi ${ }^{52}$ ", se contente en fait d'établir sur un plan général une nouvelle hiérarchie, dont il précise et limite les conséquences pratiques dans la dernière partie, en réponse sans doute à une objection de Zeuxippe. Et de même que, dans la réponse à Pemptidès, il commençait par flétrir " les injures de l'ignorance en injures féconde " contre les dieux (757 A12), de même alors, renouant avec la problématique de Daphnée, mais en tenant compte de l'inversion opérée dans le discours central, il se demande avec indignation « qui pourrait supporter que l'on injurie Aphrodite en prétendant qu'elle empêche la philia de naître si elle s'adjoint à Éros et se tient à ses côtés ? " $\left(768 \mathrm{E} 1-3^{53}\right)$. Si le telos de l'amour ne se confond évidemment pas avec les aphrodisia - le texte indique luimême que le but ultime, pour Plutarque comme pour Protogène, demeure la philia, l'union spirituelle-, Aphrodite ne saurait être considérée comme une présence parasite, elle est un point de départ et un adjuvant, à partir duquel peut s'épanouir l'intimité conjugale - mais Plutarque ne va pas jusqu'à affirmer, comme Daphnée, la nécessité de sa présence et il reconnaîtra un peu plus loin la possibilité pour l'amour des garçons de faire naître des couples durables, tout en en soulignant la rareté (770 C).

Mais Aphrodite, au rebours d'Arès, n'a pas qu'une dimension éthique : elle entre aussi dans les considérations sur l'ancienneté d'Éros, liée à sa dimension cosmique. Cette dimension, aux origines du monde, est aussi au principe de l'exposé : il faut

Plus précisément, Plutarque ne le nomme pas, mais utilise un vers tragique qui suggère un dieu et

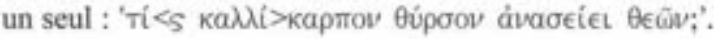

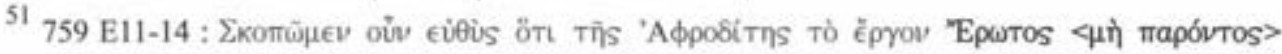

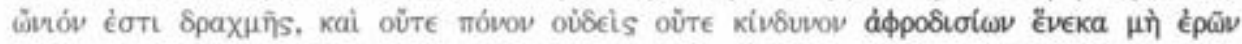

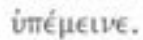

De comm. not. 1073 C, et n. 411 de D. BABUT (CUF, t. XV-2).

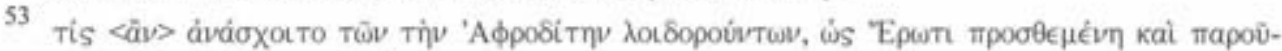

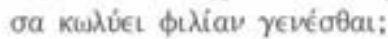


donc revenir au début de la réponse à Pemptidès et reprendre la lecture après le passage, cité plus haut, qui, tout en réduisant l'ergon d'Aphrodite à un parergon, continuait de donner grammaticalement au dieu la fonction d'instrument de la déesse. Plutarque se réfêre alors à Parménide et Hésiode, que cite aussi Phèdre dans le Banquet $^{54}$, et, derechef, il procède à une inversion, toujours dans le même dessein d'exalter l'importance d'Éros. Le texte de Parménide, cité en second par Phèdre, est donc présenté en premier, comme « montrant en Éros la plus ancienne des œuvres d'Aphrodite ", laquelle est aussi considérée par Plutarque comme le sujet du vers

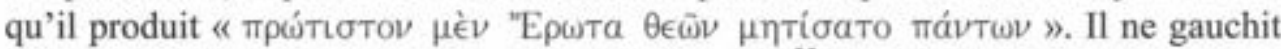
pas ainsi volontairement un texte d'ailleurs obscur ${ }^{55}:$ il donne l'interprétation qui est la sienne, comme l'atteste le De facie, où Lamprias énumère la philotès d'Empédocle, l'Aphrodite de Parménide et l'Éros d'Hésiode comme autant de désignations de la force créatrice à l'œuvre dans la nature, qu'il appelle tò i $\mu \in \rho$ Tó $v^{56}$. Mais, s'il pense que Parménide accorde à Aphrodite la primauté, il lui fait succéder

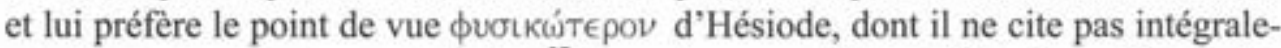
ment le texte, au rebours de Platon ${ }^{57}$, ce qui a pour effet de faire disparaître toutes les autres divinités primordiales pour ne laisser qu'Éros, qualifié de $\pi a ́ \nu \tau \omega \nu \pi \rho 0-$

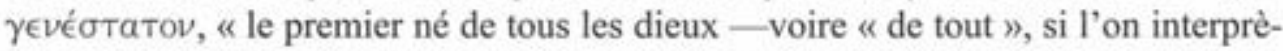
te $\pi a ́ v T \omega \nu$ comme un neutre, ce qui accentuerait encore la progression par rapport à la citation précédente ${ }^{58}$ - de sorte que tout grâce à lui participe à la génération " - formule qui n'est pas dépourvue d'une certaine couleur platonicienne. Si l'on poursuit la comparaison avec le Banquet, on se trouve alors plutôt dans la perspec-

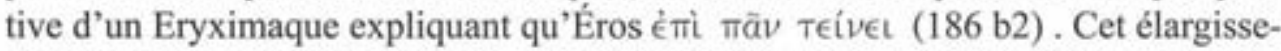
ment cosmique se retrouvera aussi dans l'exaltation finale du mariage qui culmine avec l'évocation de l'hiérogamie, oủ sont associés poètes et physikoi :

Elles sont utilisées aussi par Aristote en Metaph. I 4. 984 b23-30 : voir l'étude détaillée de MArTin, 1969, dont je reprends les grandes lignes. Dans le passage précédent, Plutarque a cité un passage

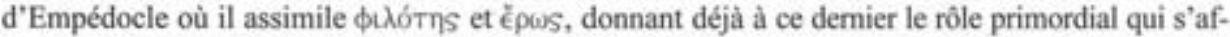
firme avec la citation d'Hésiode : voir aussi H. MAKTrN JR, « Plutarch's Citation of Empedocles at Amatorius 756 D m, GRBS, 10 (1969) 57-70.

Les incertitudes sur le sujet - sans doute la divinité féminine primordiale si l'on suit Simpliciussont exposées par MarTiN 1969, p. 189 (Simplicius), 190 (interprétation de Plutarque), 192 (Phèdre dans le Banquet), et 194 (Aristote).

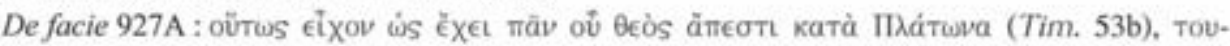

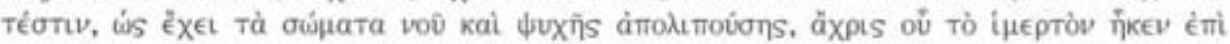

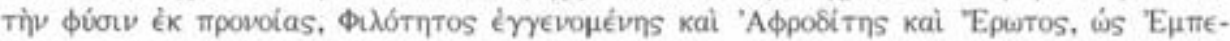

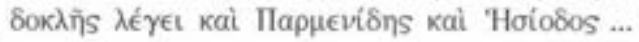

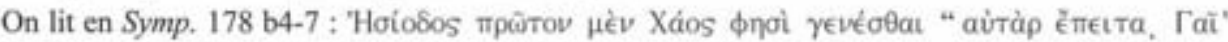

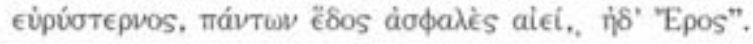

Et aussi par rapport à la citation d'Empédocle, qui permettait seulement de situer Éros év Toĩs

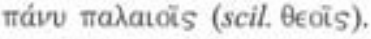


C'est ainsi que

La terre a pour la pluie un amoureux désir ( $€$ $\rho a ̄ \nu)$, disent les poètes, et le Ciel pour la Terre ; le soleil a pour la lune un amoureux désir, selon les spécialistes de la nature, et s'unit à elle pour la féconder. Et puisque la Terre est la mère des hommes et l'origine de tous les animaux et de toutes les plantes, n'est-il pas fatal qu'elle périsse et s'éteigne totalement, le jour où le puissant amour ou désir du dieu aura abandonné la matière et où elle aura cessé de désirer et de poursuivre le principe et le mouve-

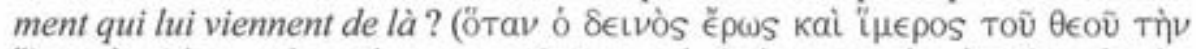

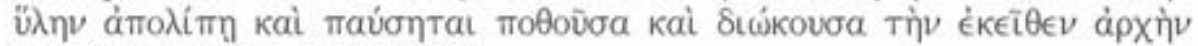

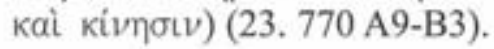

Aux poètes et physikoi s'ajoutent dans la question finale, qu'il faut sans doute

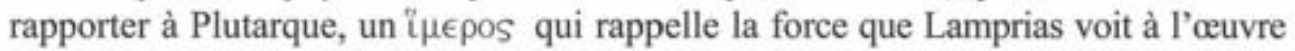
dans le De facie pour apporter aux éléments désordonnés harmonia et koinônia, et une allusion au désir de la matière qui correspond à l'interprétation de la quête d'Isis dans le Sur Isis ${ }^{59}$. Si notre passage n'est certainement pas au niveau philosophique de ces deux autres cuvres, pas plus que les références à Hésiode et Parménide ne sauraient se comparer en rien à l'utilisation qu'Aristote fait des mêmes textes dans la Métaphysique pour réfléchir au principe premier ${ }^{60}$, il apparaît cependant à Plutarque qu'il s'engage sur un terrain qui n'est plus tout à fait celui du Dialogue sur l'Amour et il coupe d'un « Mais je crains de paraître m'égarer trop loin ou même bavarder à tort et à travers » (770 B4-5), qui fait écho aux autres ruptures à l'intérieur de la réponse à Pemptidès, lorsqu'il abordait des questions philosophiques platoniciennes ${ }^{61}$ : ce fait nous ramène, en conclusion, au problème de la nature même de notre texte qu'a soulevé l'emploi des thèmes rhétoriques et des figures mythologiques.

\section{Conclusion}

Si la réponse à Pemptidès ne se veut pas un exposé philosophique et correspondrait, mutatis mutandis, à la deuxième partie d'un cours, celle où l'on répond à des questions plus pratiques, plutôt qu'à la première, consacrée à l'exégèse des grands textes, c'est ne pas en mesurer suffisamment la portée que de la réduire à un éloge en forme rhétorique et il faut, je crois, se fier à ce que dit Plutarque lui-même sans l'attribuer à un pur désir d'anxèsis : l'interrogation met en cause des éléments essentiels de

59

Voir le passage du De Iside cité à la note 31 et le commentaire de Görgemanns 2006, n. 429, p. 185.

60

oir supra n. 54.

61 17. 762 A11 et 18. 763 F7, qui entrainent les reproches de Soclaros en 19. 764 A1-4; Plutarque interrompra néanmoins aussi son hieros logos en 20.766 B13-C1, et l'on peut ajouter à la liste la prudente introduction de la théorie des maniai en 16. 758 D8-9. 
notre vie sur terre, de notre relation au divin, de la part de divin qu'il y a dans cette vie et dont la patrios pistis éveille en nous le sentiment. Cette défense de la patrios pistis est la première raison d'intégrer les dieux traditionnels, mais l'ensemble du passage ne se limite pas à cela et donne une vísion beaucoup plus complète de l'existence terrestre et du rôle qu'y tient l'amour, pour laquelle Plutarque puise abondamment dans les éloges du Banquet. En se centrant sur les deux puissances d'Arès et d'Aphrodite, il met l'accent d'abord en introduction sur la dimension psychique, avant de développer les implications éthiques à travers toute une série d'illustrations, citations et anecdotes, qui culminent dans la confrontation d'Hadès et d'Éros ${ }^{62}$.

Se dessine ainsi la figure d'Éros dans le monde de la genesis, puissance cosmique de vie, puissance psychique qui donne tous les courages, comme le montre la confrontation avec Arès, et qui s'élève aussi bien au-dessus du simple désir charnel, comme le marque la nouvelle hiérarchie établie avec Aphrodite. En redistribuant ainsi les rôles d'Éros et d'Aphrodite, Plutarque n'a plus besoin de la distinction introduite dans le Banquet par Pausanias entre Aphrodite et Éros pandémiens d'une part, Aphrodite et Éros célestes de l'autre ${ }^{63}$ : absence remarquable, tant les auteurs usent et abusent de ce topos, qu'on trouve dans l'aimable badinage des Amours du corpus de Lucien comme dans le profond traité 50 de Plotin (Enn. III 5), dans le débat très leste d'Achille Tatius comme dans les conférences sérieuses de Maxime de Tyr. L'analyse ne se focalise pas, dans cette partie, sur l'opposition du corps et de l'âme ${ }^{64}$, mais envisage le double niveau, du monde et de l'âme humaine. Dans les deux domaines, Éros exerce une force bienfaisante, qui est force de vie et d'harmonie et se fait force d'élévation dans l'âme, ce que suggère déjà la réponse à Pemptidès bien qu'elle reste située au niveau terrestre et qu'accentuera le hieros logos platonicien, le seul passage où l'on voie apparaître « l'Éros céleste » (765 B1), un Éros cependant qui n'est pas différent d'un autre, terrestre, mais qui exerce alors sa fonction la plus haute en réfractant la pensée des amants vers la Beauté véritable.

1) Textes anciens

BIBLIOGRAPHIE

BABUT, D.,

- Plutarque, De la vertu éthique, Paris, 1969.

DILLON, J.,

- ALCINOOS, The Handbook of Platonism, Oxford, 1995.

FLACELIĖRE, R.,

- Plutarque, Dialogue sur l'Amour in CEuvres Morales X, Paris, CUF, 1980.

62

D'une certaine manière, la partie sur les bienfaits reprend les mêmes thèmes en chiasme: métamorphose morale due à l'amour d'abord (17.762 B-18.762 E), puis possession de l'âme ensuite (18.762 F-763 B), qui prépare la partie plus proprement platonicienne.

63

64 Opposition qu'il transpose à la poésie dans sa critique de la pantomime, $Q C$ IX 15, $748 \mathrm{D}$.

La distinction structure au contraire la partie platonicienne, où l'influence du Phédon se fait sentir : voir FRAZIER 1999. 
GÖRGEMANNS, H.,

- PlutaRCh. Dialog über die Liebe, eingeleitet und übersetzt von H. G, mit interpretierenden Essays von B. Feichtinger, F. Graf, W. Jeanrond und J. Opsomer, SAPERE X, Tübingen, 2006.

GOTTELAND, S., \& OUDOT, E.,

- Plutarque, Dialogue sur l'Amour, Paris, GF, 2006.

VALVERDE SÁNCHEZ, M.,

- Plutarco, Erótico, in Obras Morales y de Costumbres X, BCG 309, Madrid, 2003.

WHITTAKER, J., \& LOUIS, P.,

- ALCINOos, Enseignement des doctrines de Platon, Paris, CUF, 1990.

2) Études

BARIGAZZI, A.,

- "Note critiche ed esegetiche all'Eroticos di Plutarco», Prometheus, 12 (1986) 97-122 et 245-266.

DILLON, J.,

- The Middle Platonists, London, 1977.

FrAZIER, F.,

- "Platonisme et Patrios pistis dans le discours central de l'Érotikos (chs 13-20)», in A. Pérez Jiménez, J. Garcia López y Rosa Maria Aguilar, Plutarco, Platón y Aristóteles. Actas del V Congresso Internacional de la I. P. S. (Madrid-Cuenca, 4-7 de Mayo de 1999), Madrid, 1999, 343-356.

- "La "prouesse de Camma" et la fonction des exempla dans le Dialogue Sur l'Amoum, in A. Pérez-Jiménez \& F. Titchener (edd.), Historical and Biographical Values of Plutarch's Works. Studies devoted to Professor Philip Stadter by the International Plutarch Society, 2005, 197-212 (=2005a).

- "Göttlichkeit und Glaube. Persönliche Gottesbeziehung im Spätwerk Plutarchs», in R. Hirsch-Luipold (hgs), Gott und die Götter bei Plutarch. Götterbilder -Gottesbilder Weltbilder, Berlin-New York, 2005, 111-137 (=2005b).

- "L'Érotikos : un éloge du Dieu Éros ? Une relecture du dialogue de Plutarque», Ploutarchos, n. s., 3 (2005/2006) 63-102.

GORGEMANNS, H.,

- "Eros als Gott in Plutarchs "Amatorius"», in R. Hirsch-Luipold (hgs), Gott und die Götter bei Plutarch. Götterbilder - Gottesbilder - Weltbilder, Berlin - New York, 2005, 169-195.

MARTIN JR, H.,

- "Amatorius 756 E-F : Plutarch's Citation of Parmenides and Hesiod", AJPh, 90 (1969) 183-200.

OPSOMER, J.,

- «Eros in Plutarchs moralischer Psychologie», in H. Görgemanns, 2006, 208-235.

RUSSELL, D.,

- "Plutarch's Amatorius 13-18», in J. Mossman (ed.), Plutarch and his Intellectual World, London, 1997, 99-111.

SIRINELLI, J.,

- Plutarque de Chéronée. Un philosophe dans le siècle, Paris, 2000.

VALVERDE SÁNCHEZ, M.,

- "Metáforas de la guerra en el Erótico de Plutarco», Ploutarchos, n. s., 2 (2004/2005) 123-140. 


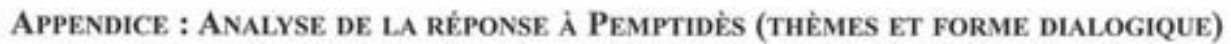

\section{Danger d'ébranler la patrios pistis et divinité d'Éros (13. 756 B-16. 759 D)}

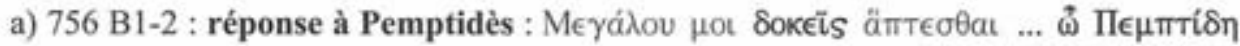
$=$ atteinte à la tradition

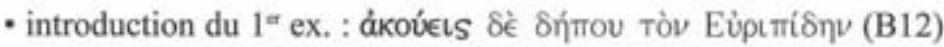

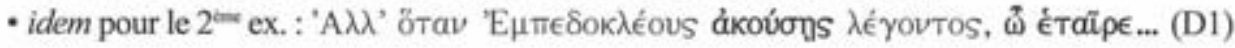

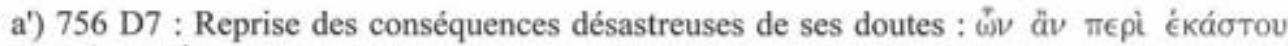

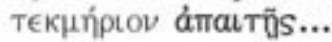

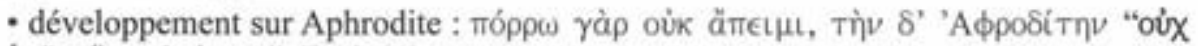

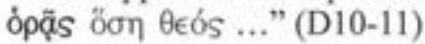

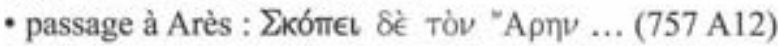

b) 757 C4-10 : dialogue entre Pemptidès et Plutarque établissant qu'il ne faut pas confondre dieu et passion, avec utilisation de l'exemple d'Arès et reprise de l'exposé par Plutarque :

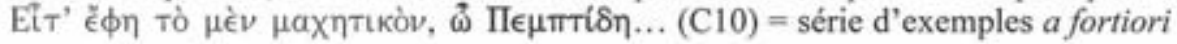

- la guerre (interrogation) ;

- la chasse (interrogation);

- la plante-homme : Plutarque se tourne vers Daphnée ( $\dot{\omega} \phi(\lambda \in \Delta a \phi v a \bar{\epsilon} \in$, E6), qui lui répond $(\mathrm{E} 8)$;

- les nécessités de la vie (introduction interrogative) : Plutarque se tourne vers

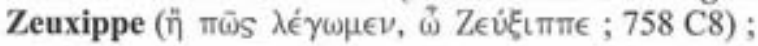

- dialogue entre Zeuxippe et Plutarque (C9-D7), pour introduire l'exemple des philiai

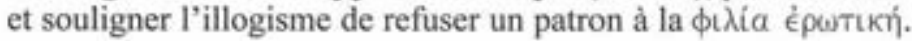

- les maniai : introduction de la théorie et énumération, avec, au moment d'arriver à Éros, prise à témoin de Daphnée ( $(\hat{\omega} \Delta a \phi v a i ̄ \epsilon, 759 \mathrm{~A} 2)$ et question à Pemptidès (ưrrè $\rho$

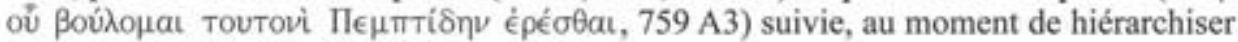
ces maniai, de ர̃ jà oủx ó

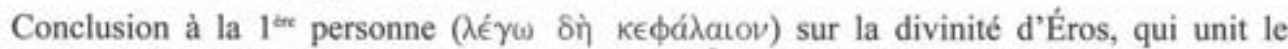

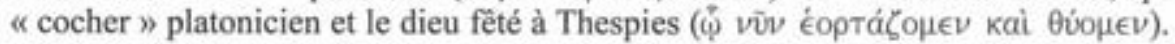

2. La puissance d'Éros (759 D9-762 A10)

Introduction de la division (759 D9-E10)

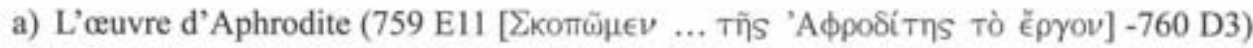

- absence de toute valeur d'Aphrodite sans Éros : vil prix des prostituées ou prostitution

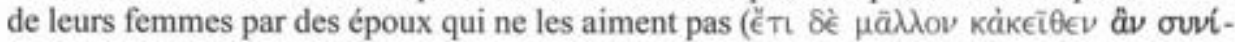
Sots, 759 F8)

- valeur d'Éros sans Aphrodite : les érastes prêts à affronter les tyrans ( $\mathrm{A} \rho \rho^{\prime}$ oũv... ớ $\theta a$; 760 B8)

- valeur d'Éros reconnue par Alexandre lui-même.

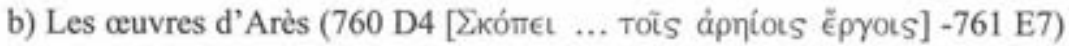

- exemples de courage sur le champ de bataille, dont 


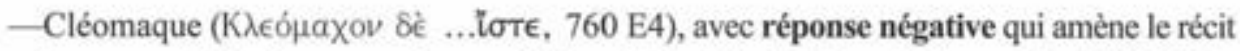

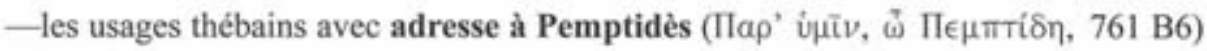

- élargissement aux peuples et héros : ex. d'Alceste (- Éros sans Arès ; plus fort que la mort)

b') Hadès dans le prolongement (761 E8-762 A10) et horizon platonicien

\section{Les bienfaits d'Éros (762 A11-763 B9)}

Rupture du thème platoncien ( $\Delta$ tò TaũTa $\mu$ èv mant (762 A11-B4)

a) La transformation morale de l'amant (17.762 B-18.762 E)

- correction d'Euripide et de son affirmation « l'Amour rend poète »

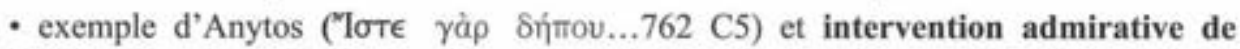
Zeuxippe

- reprise par Plutarque sur la philanthropia (interrogation), puis adresse à Daphnée (屯ี $\Delta a \phi$ vaīe, 762 E5) sur l'ascendant d'Éros dans l'âme, qui fait transition avec

b) Le bouleversement amoureux (18.762 F-763 B)

- référence à Sapho et demande à Daphnée de réciter le poème ( $\dot{\omega} \Delta$ a

- retour en commentaire du thème de la mania (interrogation) et critique de Ménandre.

Conclusion (18.763 B-763 F)

Introduction qui renvoie au début de l'intervention : ce qui aurait dû être dit alors

La theologia tripertita = accord exceptionnel de toute la tradition 


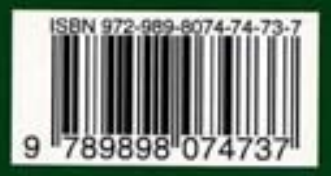

